\title{
A Designed Protein Interface That Blocks Fibril Formation
}

\author{
Ushma J. Shukla, ${ }^{\dagger}$ Heather Marino, ${ }^{\dagger}$ Po-Ssu Huang, ${ }^{\ddagger}$ Stephen L. Mayo, ${ }^{\ddagger}$ and John J. Love ${ }^{*} \dagger$ \\ Department of Chemistry, San Diego State University, 5500 Campanile Drive, San Diego, California 92182-1030, \\ and Howard Hughes Medical Institute and Divisions of Biology and Chemistry, California Institute of Technology, \\ 114-96, 1200 E. California Blvd., Pasadena, California 91125
}

Received July 19, 2004; E-mail: jlove@sciences.sdsu.edu

Almost all cellular processes entail the precise interactions of proteins that self-assemble either transiently or permanently as obligate multimers. Unlike native complexes, some proteins assemble erroneously, resulting in amyloid fibrils that form insoluble plaques known to be associated with neurodegenerative diseases, e.g., Alzheimer's disease, Parkinson's disease, bovine spongiform encephalopathy, and other prion diseases. Due to the prevalence of fibrils in these diseases, much attention has been given in recent years to development of inhibitors of protein-based fibril formation. For example, small-molecule inhibitors of transthyretin fibrillation have been designed, ${ }^{1}$ the antibiotic rifampicin causes fibril disassembly in vitro, ${ }^{2}$ and $\mathrm{C}$-reactive protein has been shown to break down amyloid deposits in the body. ${ }^{3}$ In addition, Dumoulin et al. recently developed a camelid antibody that inhibits fibrillation of lysozyme. ${ }^{4}$

Previously we engineered a de novo protein interface by first docking the normally monomeric $\beta 1$ domain of Streptococcal protein-G to itself and then mutating specific interfacial side chains so as to drive complex formation..$^{5}$ This design resulted in a pair of protein monomers that formed a heterodimer of modest binding affinity. Introduction of the specific mutations that resulted in the unique sequences of each monomer (referred to as monomer- $\mathrm{A}$ and monomer-B) altered their thermodynamic properties relative to the wild-type protein-G. The 12 mutations that resulted in the monomer-A sequence stabilized it to a hyperthermophile (i.e., $T_{\mathrm{m}}>100$ ${ }^{\circ} \mathrm{C}$ ), while the 8 for monomer-B were destabilizing, resulting in a $T_{\mathrm{m}}$ of $\sim 37{ }^{\circ} \mathrm{C}$. The $T_{\mathrm{m}}$ for WT protein-G is $\sim 83{ }^{\circ} \mathrm{C}$.

At the concentrations required for NMR studies, monomer-B alone was observed to form macroscopic fibers (Figure 1). Although interesting, this in itself is not unusual since many proteins, upon destabilization, can be induced to form fibers. ${ }^{6-11}$ The distinctive aspect of this system is that, in the presence of an equimolar quantity of monomer-A, no monomer-B fibers were observed. Thus, this engineered complex proved to be an excellent model system for studying protein-based fibril inhibition. Here we report the amyloidlike properties of the monomer-B fibrils and the ability of the monomer-A binding partner to completely block formation of monomer-B fibrils.

The physical features and chemical characteristics of amyloid fibrils were elucidated as early as 1959, when it was determined using electron microscopy that amyloid plaques do not consist of amorphous aggregates but instead are made up of microscopic fibrils. The fibrils are consistently long, straight, and unbranched with a diameter of $\sim 10 \mathrm{~nm} .{ }^{12}$ All amyloid fibrils exhibit cross- $\beta$ fibril diffraction and consist of the same structure that contains a $\beta$-sheet oriented perpendicular to the axis of the fibril. ${ }^{13}$ They also cause a significant shift and marked increase in the fluorescence of the dye Thioflavin T (ThT). ${ }^{14}$ Transmission electron microscopy (TEM) and ThT fluorescence were used to characterize the amyloid-

\footnotetext{
$\dagger$ San Diego State University.

\# California Institute of Technology.
}

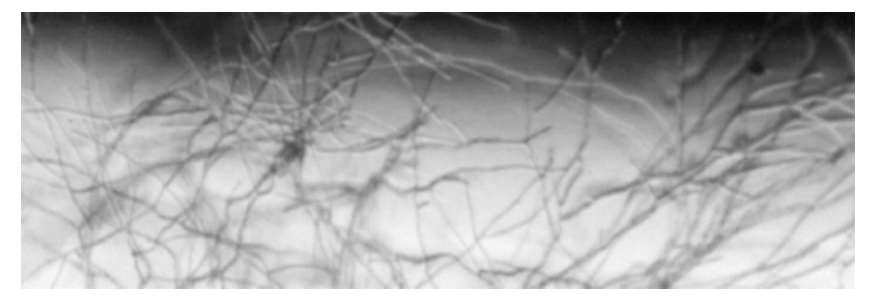

Figure 1. Macroscopic fibers were observed to form spontaneously after approximately 3 days in an NMR tube that contained free monomer-B.

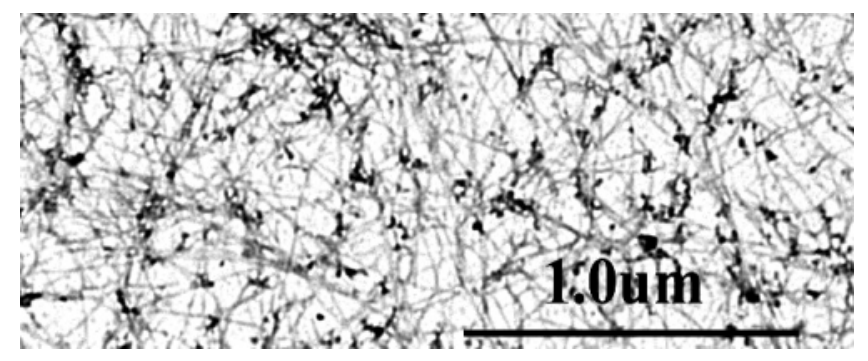

Figure 2. Transmission electron micrograph of monomer-B fibrils. Although densely packed, the individual microscopic fibrils are unbranched and of indeterminate length. The diameter of the fibrils is approximately $10 \mathrm{~nm}$.

like properties of the monomer-B fibrils and to confirm the inhibitory properties of the binding partner monomer-A.

Monomer-B fibrils were subjected to TEM imaging to confirm that the fibrils formed were indeed amyloid-like. The micrograph reveals that monomer-B forms long unbranched fibrils with a diameter of approximately $10 \mathrm{~nm}$ (Figure 2). These parameters are consistent with the morphology of amyloid type fibrils. ${ }^{15}$

To quantify the amount of fibrils formed, the fluorescent dye ThT was utilized. The ThT fluorescence signal shows a large shift and increase upon binding to $\beta$-sheet-containing fibrils. ${ }^{14}$ To fully characterize the inhibitory properties of monomer-A, it was necessary to achieve consistent fibrillation of monomer-B. Monomer$\mathrm{B}$, at concentrations used for NMR analysis (i.e., $\sim 1.25 \mathrm{mM}$ ), was incubated at four temperatures: $4,18,25$, and $37^{\circ} \mathrm{C}$. Fibrils were visible in the 25 and $37{ }^{\circ} \mathrm{C}$ samples after 5-7 days. No fibrils were observed at the other temperatures.

Agitation at the $T_{\mathrm{m}}$ of a protein has been used in fibril formation studies as it shortens the time required for fibrillation. ${ }^{16}$ RamirezAlvarado et al. demonstrated that agitation and incubation at the $T_{\mathrm{m}}$ of a number of protein-G mutant variants shortened the onset of fibrillation. ${ }^{8}$ Samples were incubated at 25 and $37{ }^{\circ} \mathrm{C}$ and were agitated and observed visually. After 3 days, more fibrils were observed in the $37^{\circ} \mathrm{C}$ sample; therefore, all subsequent experiments were performed at $37{ }^{\circ} \mathrm{C}$ with agitation.

In addition to agitation, it has also been shown that incubation with seed fibrils hastens the onset and consistency of fibril formation. ${ }^{8,17}$ To normalize seed fibrils added to the fibril formation solutions, $1.25 \mathrm{mM}$ concentrations of preformed monomer-B fibrils 


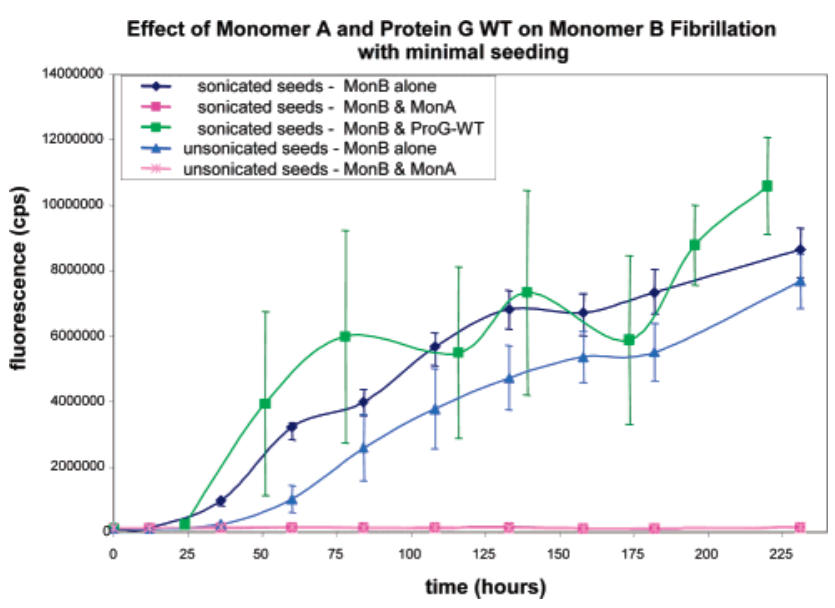

Figure 3. ThT fluorescence plotted against incubation time. Monomer-A blocked monomer-B fibrillation, as confirmed by the fact that no increase in ThT fluorescence was observed for monomer-B fibrils in the presence of monomer-A (light and dark pink, flat lines). In contrast, samples of free monomer-B that received sonicated (dark blue) and unsonicated (lighter blue) seed fibrils showed a marked increase in ThT fluorescence after approximately 24 and $48 \mathrm{~h}$, respectively. WT protein-G was incubated with monomer-B and resulted in an increase in ThT fluorescence (green).

were sonicated with three 10-s pulses in a water bath sonicator. Dilutions of 1:100, 1:1000, and 1:10000 of the sonicated seed fibrils were used in the onset of fibrillation analysis. Fibril induction was run in triplicate on $0.6 \mathrm{mM}$ monomer-B solutions. On the basis of the results, it was determined that the 1:1000-fold dilution was most consistent in the onset of fibril formation, and therefore both sonicated and unsonicated 1:1000-fold dilution of seed fibrils were used for the inhibition assays.

To study the inhibitory effect of monomer-A on monomer-B fibril formation, triplicate solutions of equimolar quantities (i.e., $0.6 \mathrm{mM}$ ) of the two binding partners were incubated at $37{ }^{\circ} \mathrm{C}$ with agitation and in the presence of seed fibrils for approximately 9 days (Figure 3). In both cases, the ThT fluorescence did not increase and therefore confirmed that the presence of monomer-A completely blocked the ability of monomer-B to form fibrils. As a positive control, monomer-B alone was incubated as above in the presence of both sonicated and unsonicated 1:1000-fold dilution of seed fibrils. As predicted, the monomer-B alone samples formed fibrils and caused a significant increase in ThT fluorescence after only 1.5 days (Figure 3 ). The samples that received the sonicated fibrils exhibited a more rapid increase in signal intensity, reflecting an increase in fibril formation. As a negative control, solutions of 0.6 $\mathrm{mM}$ monomer-A alone were incubated at $37^{\circ} \mathrm{C}$ with agitation and also in the presence of preformed monomer-B fibrils under identical conditions. In both sets of experiments, no fibrils were observed (data not shown).

To explore the specificity of monomer-A inhibition, equimolar quantities (i.e., $0.6 \mathrm{mM}$ ) of WT protein-G and monomer-B were incubated under the conditions described above. WT protein- $\mathrm{G}$ is a structural homologue of monomer-A; however, unlike monomer$\mathrm{A}$, it was not engineered to interact with monomer-B, and thus should not inhibit monomer-B fibrillation. As expected, when monomer-B is agitated in the presence of an equimolar quantity of WT protein-G, fibril formation is not blocked, yet interestingly, this solution exhibited a greater increase in ThT fluorescence than the monomer-B samples alone. Since Ramirez-Alvarado et al. have demonstrated cross-seeding abilities for some of their protein-G mutants, we incubated WT protein-G alone with preformed monomer-B seed fibrils and monitored ThT fluorescence. After more than 2 weeks, no fibrils were observed. This finding implies that it is not likely that the increase in fluorescence observed is due to cross-seeding. Since WT protein-G alone does not form fibrils at $37{ }^{\circ} \mathrm{C}$, even in the presence of preformed monomer-B fibrils, its presence may be affecting monomer-B fibril formation by another mechanism. It has been demonstrated experimentally that molecular crowding, induced by the addition of high concentrations of different polymers, can dramatically accelerate $\alpha$-synuclein fibrillation in vitro. ${ }^{18}$ If molecular crowding, induced by the equimolar presence of WT protein-G, is the cause of the increase in ThT fluorescence, then the ability of monomer-A to completely block monomer-B fibril formation is of even greater significance, as it occurs despite a concomitant increase in molecular crowding.

We have demonstrated the ability of a destabilized test protein, monomer-B, to consistently form amyloid fibrils and, more importantly, proved that a designed protein interface can effectively block protein fibril formation. We hypothesize that, in a manner similar to that observed for the camelid antibody that inhibits fibrillation of lysozyme, ${ }^{4}$ monomer-A functions to bind and stabilize the target fold of monomer- $\mathrm{B}$ in the engineered heterodimer. Complex formation may effectively sequester and stabilize monomer-B and prevent it from sampling folding intermediates that give rise to self-assembled fibrils. In addition, inhibition by the designed binding partner is specific in that a structurally homologous variant (WT protein-G) did not block monomer-B fibril formation and actually increased ThT fluorescence. Work is underway to apply these protein design/fibril inhibition techniques to proteins known to cause disease upon fibrillation. In addition, we are considering the use of this system to alter the physical characteristics of the fibrils in an attempt to generate nanostructures of specific geometries. ${ }^{19,20}$

Acknowledgment is made for support of this research to the donors of the American Chemical Society Petroleum Research Fund, the Blasker-Rose-Miah fund of the San Diego Foundation, and the California Metabolic Research Foundation.

\section{References}

(1) Klabunde, T.; Petrassi, H. M.; Oza, V. B.; Raman, P.; Kelly, J. W.; Sacchettini, J. C. Nat. Struct. Biol. 2000, 7, 312-321.

(2) Findeis, M. A. Biochim. Biophys. Acta 2000, 1502, 76-84

(3) Pepys, M. B.; Hirschfield, G. M. J. Clin. Invest. 2003, 111, 1805-1812.

(4) Dumoulin, M.; Last, A. M.; Desmyter, A.; Decanniere, K.; Canet, D.; Larsson, G.; Spencer, A.; Archer, D. B.; Sasse, J.; Muyldermans, S.; Wyns, L.; Redfield, C.; Matagne, A.; Robinson, C. V.; Dobson, C. M. Nature 2003, 424, 783-788.

(5) Love, J. J.; Huang, P.-S.; Mayo, S. L. Manuscript in preparation

(6) Lee, S.; Eisenberg, D. Nat. Struct. Biol. 2003, 10, 725-730.

(7) Morozova-Roche, L. A.; Zurdo, J.; Spencer, A.; Noppe, W.; Receveur, V.; Archer, D. B.; Joniau, M.; Dobson, C. M. J. Struct. Biol. 2000, 130, 339-351.

(8) Ramirez-Alvarado, M.; Regan, L. J. Mol. Biol. 2002, 323, 17-22.

(9) Kheterpal, I.; Williams, A.; Murphy, C.; Bledsoe, B.; Wetzel, R Biochemistry 2001, 40, 11757-11767.

(10) Khurana, R.; Gillespie, J. R.; Talapatra, A.; Minert, L. J.; Ionescu-Zanetti, C.; Millett, I.; Fink, A. L. Biochemistry 2001, 40, 3525-3535.

(11) West, M. W.; Wang, W.; Patterson, J.; Mancias, J. D.; Beasley, J. R.; Hecht, M. H. Proc. Natl. Acad. Sci. U.S.A. 1999, 96, 11211-11216.

(12) Cohen, A. S.; Calkins, E. Nature 1959, 183, 1202-1203.

(13) Jimenez, J. L.; Guijarro, J. I.; Orlova, E.; Zurdo, J.; Dobson, C. M.; Sunde, M.; Saibil, H. R. Embo J. 1999, 18, 815-821.

(14) LeVine, H., III. Methods Enzymol. 1999, 309, 274-284.

(15) Nielsen, E. H.; Nybo, M.; Svehag, S. E. Methods Enzymol. 1999, 309, 491-496.

(16) Nielsen, L.; Khurana, R.; Coats, A.; Frokjaer, S.; Brange, J.; Vyas, S.; Uversky, V. N.; Fink, A. L. Biochemistry 2001, 40, 6036-6046.

(17) O'Nuallain, B.; Williams, A. D.; Westermark, P.; Wetzel, R. J. Biol. Chem. 2004, 279, 17490-17499.

(18) Uversky, V. N.; E, M. C.; Bower, K. S.; Li, J.; Fink, A. L. FEBS Lett. 2002, 515, 99-103.

(19) MacPhee, C. E.; Dobson, C. M. J. Am. Chem. Soc. 2000, 122, $12707-$ 12713.

(20) Lashuel, H. A.; LaBrenz, S. R.; Woo, L.; Serpell, L. C.; Kelly, J. W. J. Am. Chem. Soc. 2000, 122, 5262-5277.

JA0456858 